\title{
Comparative Study of Serum Testosterone Levels in Male Patients of Type 2 Diabetes Mellites and Non Diabetics Presenting with Complaints of Sexual Dysfunction
}

\author{
Authors \\ Dr Sandeep Chowdhary, MBBS, MD (Medicine) ${ }^{1}$, Dr Minaj Ahmad, D.N.B (Medicine) ${ }^{2}$ \\ ${ }^{1}$ Senior Consultant Physician, Dr.RML Combined Hospital, Lucknow INDIA \\ ${ }^{2}$ Dr.RML Combined Hospital, Lucknow INDIA
}

\begin{abstract}
Background: Diabetes mellitus is the frequent cause of sexual dysfunction in males. Various studies have reported 25 - $32 \%$ of men with Diabetes mellites complaining of sexual dysfunction.

Material and Methods: This was a prospective observational study, conducted among 100 men (aged 35-60 years) with type 2 diabetes INo Diabetes (50 cases each) and with complaints of sexual dysfunction, from June 2015 to and February May 2016.

Results: Mean age of diabetics was $44.5 \pm 7.9$ years and non diabetics was $42.54 \pm 5.63$ years. $56 \%$ of Diabetics and $20 \%$ non diabetics had lower levels of total testosterone.68\% of Diabetics and $26 \%$ non diabetics had lower levels of free testosterone. Mean serum free testosterone in diabetics was $3.2 \pm 0.78$ and in non diabetics was $6.2 \pm 3$. Mean serum FSH level in diabetics was $4.3 \pm 1.2$ and in non diabetics was $9 \pm$ 1.8. Mean serum LH level in diabetics was $6.4 \pm 2.1$ and in non diabetics was $12 \pm 2.8$. Mean Prolactin levels in Diabetics and non Diabetics was $10.5 \pm 2.6$ and $15 \pm 2.5$. Mean SHBG levels in Diabetics and non diabetics was $8.6 \pm 1.8$ and $16 \pm 3.2$. Prevalence of Hypogonadism was $22 \%$ in Diabetics and $10 \%$ in Non diabetics.

Conclusion: Hypogonadism is more prevalent in patients with Type 2 Diabetes mellites than non-diabetics. It is associated with low levels of free testosterone.

Keywords: Type 2 Diabetes Mellites, Sexual Dysfunction, Low serum total testosterone, Hypogonadism.
\end{abstract}

\section{Introduction}

Diabetes mellitus is estimated to be present in 300 million people worldwide. This number is expected to reach 450 million by the year 2030, with majority of all cases occurring in low- to middle-income countries. Diabetes mellitus is frequent cause of sexual dysfunction in males. Various studies have reported $25-32 \%$ of men with Diabetes mellites complaining of sexual dysfunction $^{(1)}$.
Sexual dysfunction is often associated with microangiopathy. Hence, the presence of retinopathy is usually a good indicator of sexual dysfunction. Age, duration of DM and the presence of other diabetic complications correlates more with its future development. Between the ages of 40 to 70 years, the prevalence of sexual dysfunction is $52 \%$ for the general population, whereas in age-matched diabetics, it is as high as $75 \%$. For men younger than 40 years of age, the 
prevalence is $7.8 \%$, but for men older than 40 years of age, it increases to $63 \%$ for type 1 diabetics and $71.1 \%$ for type 2 diabetics. The use of alcohol or antihypertensive medication appears to increase the risk of ED in this population ${ }^{(2)}$.

The relation between low serum testosterone and Diabetes mellitus has received substantial attention since last decade .Studies have reported that men with type 2 Diabetes mellites have a high prevalence of low serum testosterone. Further, reduced total testosterone levels have been related with insulin resistance and consequent risk for developing T2DM. The main symptoms of low serum testosterone are reduced libido, erectile dysfunction, reduced muscle mass and strength, increased adiposity, osteoporosis, depressed mood, fatigue, low energy, and impaired quality of life.

The pathophysiology of sexual dysfunction in diabetes is not well understood. Existing neuropathy or vasculopathy (microangiopathy and generalised vascular disease) is considered to be the major factors in the pathophysiology of DMinduced sexual dysfunction ${ }^{(3)}$.

Research has been increasingly directed for understanding the role of vascular endothelium and its control of penile cavernous smooth muscle tone. The two corpus cavernosum, which are the erectile bodies of the penis, has of multiple lacunar space encircled by cavernous smooth muscle ${ }^{(4)}$. These lacunar spaces are lined by the endothelium. In sexual activity, neurotransmitters (nitric oxide, the most important) are released from the penile nerve ending and the endothelium that triggers a relaxation of both cavernosal arteries and smooth muscle. This increases penile blood flow, causes dilation of the lacunar space and, eventually, an erection. In Diabetes mellites, it has been shown that endothelium-dependent smooth muscle relaxation is impaired, although the actual mechanism is not known. Endothelial dysfunction then leads to erectile dysfunction through smooth muscle dysfunction in the microvascular tree of the penis (5).
Acute hyperglycemia may also induce endothelial dysfunction and also decrease the velocity of nerve conduction. Chronic hyperglycemia is associated with the loss of myelinated and unmyelinated fibres and attenuated nerve fibre regeneration. Diffuse abnormalities of the peripheral nerve vasculature (vasa nervosum) are well documented in diabetics.In the late stages of diabetes, there may be complete vascular occlusion with atherosclerosis, thickening of capillary basement membrane, dilation and microaneurysms of capillaries as well as endothelial hyperplasia, and desquamation and degeneration of pericytes .These lesions induce a microangiopathy that leads to endoneurial hypoxia and demyelination ${ }^{(6,7)}$. The lesions decrease the blood flow to the vasonervosum and, thus, lead to nerve ischemia. A peripheral neuropathy then develops, which initially affects small unmyelinated fibres.

Researchers have highlighted the potential metabolic consequences of testosterone decline on age-associated metabolic changes such as abdominal obesity, diabetes, and markers of prediabetes. There is a growing interest in understanding the concurrence of symptoms of low testosterone and a low testosterone level since the clinical significance of a low testosterone level alone is unclear ${ }^{(8,9)}$.

TT concentrations are determined, to a large extent, by the circulating sex hormone binding globulin (SHBG) concentrations. In the blood of normal men, $44 \%$ of TT is bound to SHBG, $2 \%$ is unbound (free testosterone (FT)), and 54\% circulates bound to albumin and other proteins. It is not known whether the lower testosterone levels in diabetics are associated with changes in luteinizing hormone (LH) and follicular stimulating hormone (FSH).

In this context, present research conducted at Dr. RML Combined Hospital, Lucknow, intends to study the serum Testosterone levels in male patients of Type 2 Diabetes Mellites and non diabetics presenting with complaints of sexual dysfunction. 


\section{JMSCR Vol||05||Issue||03||Page 18910-18916||March}

\section{Material and Methods}

This was a prospective observational study, conducted among 100 men (aged 35-60 years) with type 2 diabetes and complaints of sexual dysfunction, from June 2015 to and May 2016 at Dr. RML Combined Hospital, Lucknow. The patients' demographic characteristics were collected using a pre structured questionnaire. Demographic characteristics, Duration of diabetes, smoking habits, presence of retinopathy, neurop- athy, and nephropathy were collected. All participants were asked to complete the International Index of Erectile Function questionnaire. Venous blood sample was collected to test for total testosterone (TT), free testosterone (FT), sex hormone binding globulin (SHBG), follicle-stimulating hormone (FSH), luteinizing hormone (LH), prolactin (PRL), serum lipids, and glycosylated hemoglobin (HbA1c). LST was defined as TT $<3 \mathrm{ng} / \mathrm{ml}$.

Results

Table.1 Age profile

\begin{tabular}{|l|c|c|c|}
\hline Variable & Diabetics & Non-Diabetics & P Value \\
\hline Mean Age (years) & $44.5 \pm 7.9$ & $42.54 \pm 5.63$ & 0.16 \\
\hline
\end{tabular}

Table.2 Socio-economic classification of Study group (Kuppuswamy classification )

\begin{tabular}{|l|c|c|c|}
\hline Class & Diabetics & Non-Diabetics & P Value \\
\hline Upper class & 3 & 4 & 0.86 \\
\hline Upper middle class & 5 & 6 & \\
\hline Lower middle class & 17 & 13 & \\
\hline Upper lower class & 19 & 21 & \\
\hline Lower class & 6 & 7 \\
\hline Total & 50 & 50 & \\
\hline
\end{tabular}

Table.3 Body Mass Index

\begin{tabular}{|l|c|c|c|}
\hline BMI $\left(\mathrm{Kg} / \mathrm{m}^{2}\right)$ & Diabetics & Non-Diabetics & 0.21 \\
\hline $\mathbf{1 0 . 5}$ & 0 & 0 & \\
\hline $\mathbf{1 8 . 5}-\mathbf{2 4 . 9}$ & 12 & 10 \\
\hline $\mathbf{1 8 . 5}-\mathbf{2 4 . 9}$ & 22 & 18 \\
\hline $\mathbf{2 5 . 0}-\mathbf{2 9 . 9}$ & 8 & 11 \\
\hline $\mathbf{3 0 . 0}-\mathbf{3 4 . 9}$ & 7 & 11 \\
\hline $\mathbf{3 5 . 0}-\mathbf{3 9 . 9}$ & 1 & 0 \\
\hline
\end{tabular}

Table.4 Waist - Hip Ratio

\begin{tabular}{|l|c|c|c|}
\hline Variables & Diabetics & Non-Diabetics & P Value \\
\hline Waist circumference $>\mathbf{9 0}$ cm & 6 & 4 & 0.14 \\
\hline Waist - Hip Ratio $(\mathbf{0 . 9 5})$ & 4 & 3 & \\
\hline
\end{tabular}

Table.5 Personal Habits

\begin{tabular}{|l|c|c|c|}
\hline Variables & Diabetics & Non-Diabetics & P Value \\
\hline H/o Alcohol Intake & 8 & 7 & 0.14 \\
\hline H/o Smoking & 7 & 9 & \\
\hline
\end{tabular}

Table.6 International Index of Erectile Function Score

\begin{tabular}{|l|c|c|c|}
\hline Variables & Diabetics & Non-Diabetics & P Value \\
\hline Mild erectile dysfunction & 12 & 15 & 0.07 \\
\hline Mild to moderate erectile dysfunction & 16 & 14 & \\
\hline Moderate erectile dysfunction & 18 & 16 & \\
\hline Severe erectile dysfunction & 4 & 5 & \\
\hline
\end{tabular}




\section{JMSCR Vol||05||Issue||03||Page 18910-18916||March}

Table.7 Serum gonadal hormones

\begin{tabular}{|l|c|c|c|}
\hline Hormones & Diabetics & Non-Diabetics & P value \\
\hline Total testosterone $(\mathbf{n g} / \mathbf{m l})$ & $3.2 \pm 0.78$ & $6.2 \pm 3.4$ & 0.001 \\
\hline Free testosterone $(\mathbf{p g} / \mathbf{m l})$ & $8.6 \pm 2.3$ & $46 \pm 12.1$ & 0.001 \\
\hline FSH $(\mathbf{M I U} / \mathbf{m l})$ & $4.3 \pm 1.2$ & $9 \pm 1.8$ & 0.02 \\
\hline LH $(\mathbf{M I U} / \mathbf{m l})$ & $6.4 \pm 2.1$ & $12 \pm 2.8$ & 0.03 \\
\hline Prolactin $(\mathbf{n g} / \mathbf{m l})$ & $10.5 \pm 2.6$ & $15 \pm 2.5$ & 0.04 \\
\hline SHBG(nm/L) & $8.6 \pm 1.8$ & $16 \pm 3.2$ & 0.03 \\
\hline
\end{tabular}

Diagram.1 Serum gonadal hormones

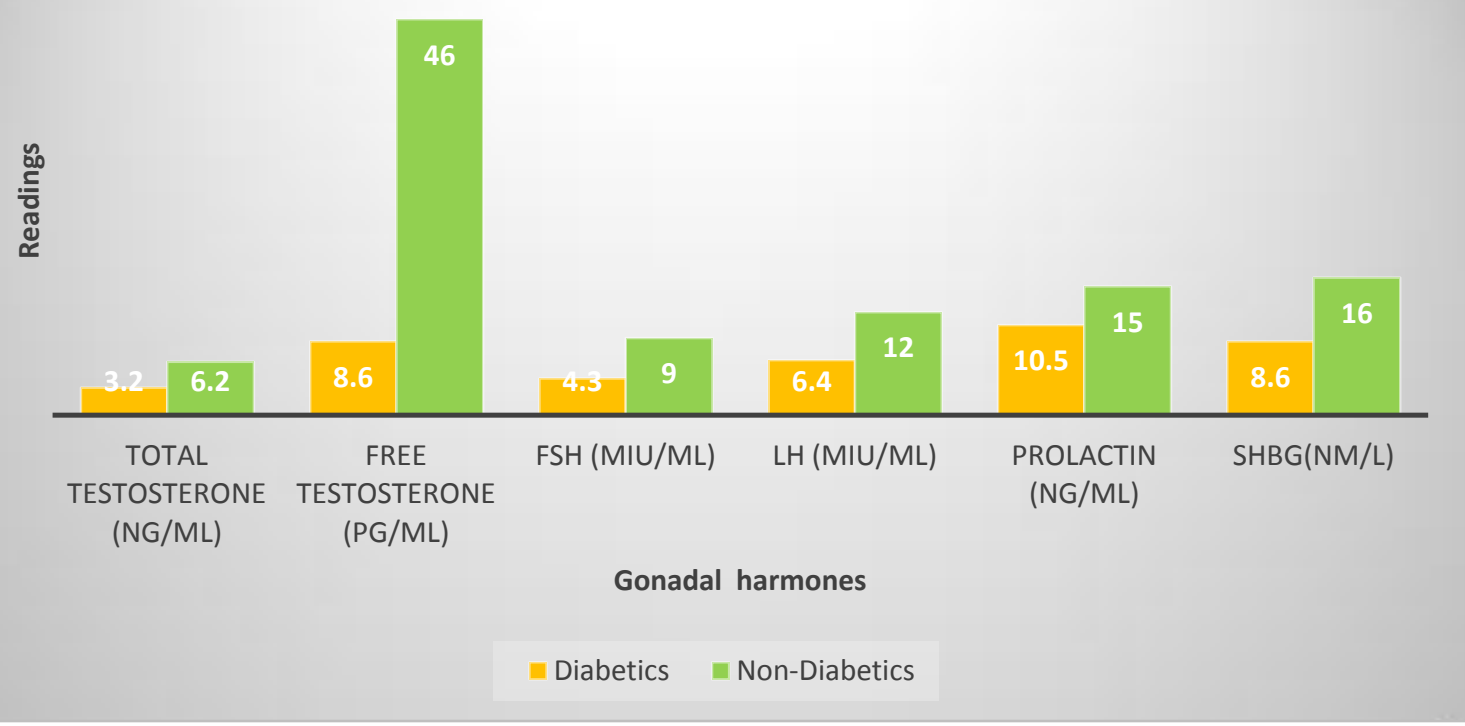

Table. 8 Incidence of Hypogonadism

\begin{tabular}{|l|c|c|c|}
\hline Variables & Diabetics & Non-Diabetics & P Value \\
\hline Hypogonadism & $11(22 \%)$ & $5(10 \%)$ & 0.02 \\
\hline Prevalence of Low Total testosterone & $28(56 \%)$ & $10(20 \%)$ & 0.04 \\
\hline Prevalence of Low free testosterone & $34(68 \%)$ & $13(26 \%)$ & 0.04 \\
\hline
\end{tabular}

Diagram. 2 Incidence of Hypogonadism

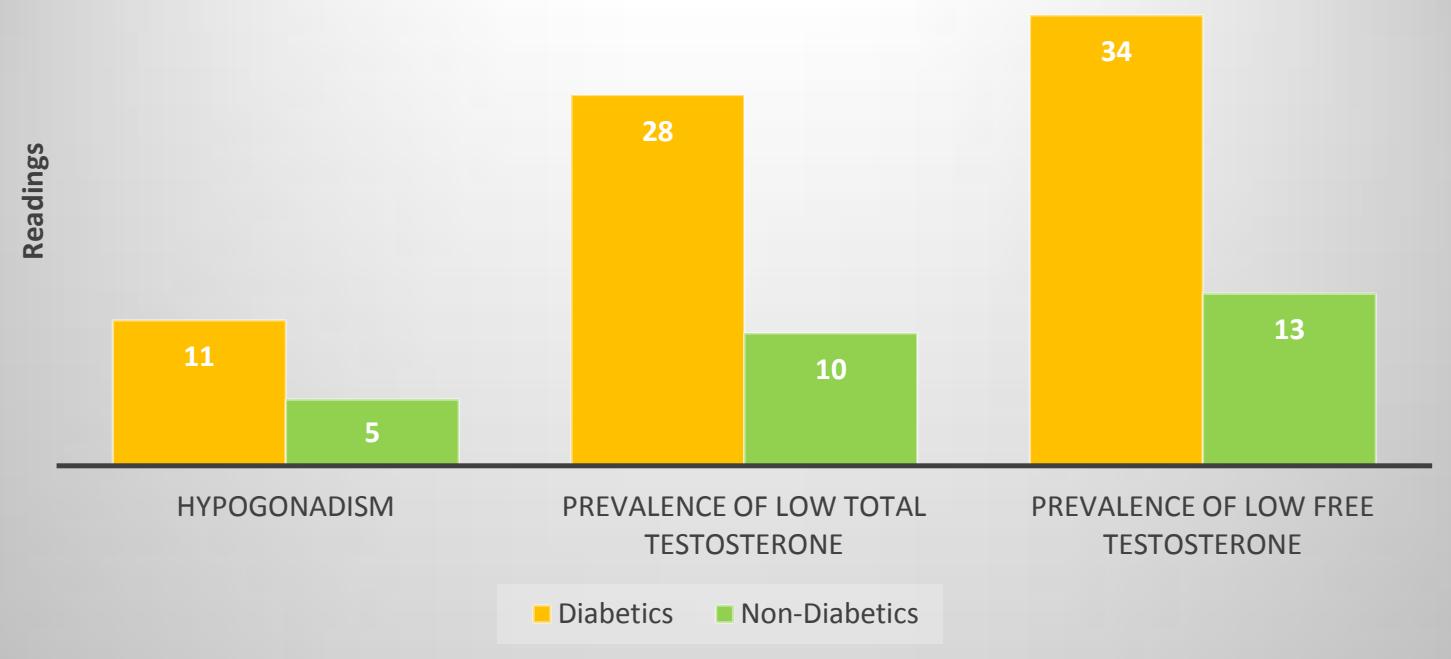




\section{Discussion}

Diabetes mellitus is most common endocrine disorder in India and worldwide which leads to significant morbidity and mortality due to accompanying complications. Erectile dysfunction, orgasmic dysfunction, reduced libido, and retrograde ejaculation are established complications found with men with long standing diabetes. Research found that patients with type 2 diabetes have higher frequency occurrence of hypogonadism ${ }^{(10)}$.

In our study, International Index of Erectile Function Questionnaire was used to elicit composite score on sexual dysfunction. Mild to Moderate erectile dysfunction was most frequent among both diabetics and non diabetics in our study.

Mean age of diabetics was $44.5 \pm 7.9$ years and non diabetics was $42.54 \pm 5.63$ years and there was no statistically difference between the age groups. Similarly, BMI and Waist to Hip Ratio was compared to between 2 groups and there was no statistically difference between the age groups. Socio-economic status was comparable with both the groups. (Kuppuswamy classification). There was no significant difference between H/o Alcohol intake and smoking between bothy the groups. In present study, $56 \%$ of Diabetics and $20 \%$ non diabetics had lower levels of total testosterone. Further, $68 \%$ of Diabetics and $26 \%$ non diabetics had lower levels of free testosterone. In a study by Ramkishan Jat et al in 2012, they found $61.51 \%$ had low levels of total testosterone and $76.9 \%$ had low serum free testosterone (11). Similarly, Dhindsaet et al in 2010 reported prevalence of low testosterone in obese diabetics to be $50 \%{ }^{(12)}$. In the study by Kapoor et al in 2007, low levels of total testosterone was found in $(25 \%)$ of patients with diabetes mellitus. In the study by Ernaniet et al in 2005,level of free testosterone was decreased in $46 \%$ of diabetics as compared to $24 \%$ in nondiabetics ${ }^{(13,14)}$.

The phenomenon of testosterone deficiency in diabetic patients is because of increase in adipose tissues mass which results in increased aromatase activity, causing enhanced conversion of testosterone in to estradiol which further suppres hypothalamic-pituitary - gonadal axis and decreases testosterone secretion. Adipose tissue, which is also considered as an endocrine organ , which a host of hormones and cytokines, can modulate insulin action and regulate leydig cell functions. Production of Leptin is tightly coupled to insulin resistance and can play a significant role in steroid biogenesis and reduced testosterone levels. Leptin levels have been shown to be inversely correlated with serum testosterone levels in various studies (Lukaa et al, 1999, Hafnear et al, 1998).

In the present study, mean serum free testosterone in cases was $3.2 \pm 0.78$ and in control group was $6.2 \pm 3$. The difference was statistically significant. In a study by Ramkishan Jat et al, mean serum free testosterone in cases was 7.65 2.04 and in control group was $11.17 \pm 2.30$. The difference was statistically significant.

Mean serum FSH level in the present study, in diabetics was $4.3 \pm 1.2$ and in non diabetics was $9 \pm$ 1.8. The difference was statistically significant. In a study by Ramkishan Jat et al, mean serum FSH level in cases was 5.82 and in control group was 6.49. The difference was statistically not significant.

In present study, mean serum LH level in cases was $6.4 \pm 2.1$ and in control group was $12 \pm 2.8$. The difference was statistically significant. In a study by Ramkishan Jat et al, mean serum LH level in cases was 5.53 and in control group was 6.22. The difference was statistically not significant.

In present study, mean Prolactin levels in Diabetics and non Diabetics was $10.5 \pm 2.6$ and 15 \pm 2.5 respectively. In study by $\mathrm{Al}$ Hayek et al, mean Prolactin levels in Diabetics and non Diabetics was $10.4 \pm 8.2$ and $18 \pm 1.5$ respectively. The difference was statistically significant in both the studies.

Mean SHBG levels in present study in Diabetics and non - diabetics was $8.6 \pm 1.8$ and $16 \pm 3.2$ respectively. In study by $\mathrm{Al}$ Hayek et al, mean 
SHBG levels in Diabetics and non Diabetics was $38.6 \pm 3.2$ and $42 \pm 4.1$ respectively. The difference was statistically significant in both the studies. The term secondary (hypogonadotropic) HG denotes an inadequate release of gonadotropinreleasing hormone $(\mathrm{GnRH})$ and is characterized by low-normal or low levels of FSH, LH, and testosterone.

In our study, prevalence of Hypogonadism was $22 \%$ in Diabetics and $10 \%$ in Non diabetics. In a prospective cross study by Datta et al on 354 men with type 2 diabetis mellites aged showed that $17 \%$ had hypogonadism with Testosterone levels $<8 \mathrm{nmol} / 1$ and a further $26 \%$ had symptoms of hypogonadism associated with a testosterone level between 8 and $12 \mathrm{nmol} / \mathrm{l}$. In a recent study from Egypt reported 32.2\% hypogonadism in type 2 diabetes patients.In another study from Brazil showed that Free testosterone and Total testosterone levels were subnormal in 46 and 34\% of diabetics, respectively.

DM is frequently associated with microvascular complications such as nephropathy, retinopathy and neuropathy with end organ damage in $50 \%$ people with the disease. This explains the possible mechanism of hypogonadism which can be extension of micro and macro vascular damage. This was highlighted in numerous studies which found that a low total serum testosterone level was associated with diabetic neuropathic patients.

\section{Conclusion}

Hypogonadism is more prevalent in Type2 Diabetes mellites patients than non-diabetics. It is associated with low levels of free testosterone. As the India harbours largest number of diabetics in the world, we recommend implementation of early universal screening programs, irrespective of the symptoms of androgen deficiency, in order to detect those with a low total serum testosterone level at an early stage and supplement testosterone accordingly. We also recommend the screening of all type 2 diabetics for androgen levels in healthcare settings for referral to endocrineologists.
Limitations of the study: Study included only Type 2 Diabetic patients and Type 1 diabetics were excluded.

\section{Financial support and sponsorship: Nil \\ Conflicts of interest: There are no conflicts of interest}

\section{Bibliography}

1. Tripathy D, Dhindsa S, Garg R, Khaishagi A, Syed T, Dandona P. Hypogonadotropic hypogonadism in erectile dysfunction associated with type 2 diabetes mellitus: A common defect? Metab Syndr Relat Disord. 2003;1:75-80.

2. Morales A. Androgens are fundamental in the maintenance of male sexual health. CurrUrol Rep. 2011;12(6):453-460

3. Gross JL, de Azevedo MJ, Silveiro SP, Canani LH, Caramori ML, Zelmanovitz T. Diabetic nephropathy: Diagnosis, prevention, and treatment. Diabetes Care. 2005; 28:164-76.

4. Cai X, Tian Y, Wu T, Cao CX, Li H, Wang KJ. Metabolic effects of testosterone replacement therapy on hypogonadal men with type 2 diabetes mellitus: a systematic review and meta-analysis of randomized controlled trials. Asian J Androl. 2014;16(1):146-152.

5. Shaheen DA, Shaheen DA, Habib AA, Ali AME. The effect of serum free testosterone level on glycemic control and atherosclerosis in type 2 diabetic men. AdvBiochem. 2014;2(2):34-39.

6. Wilkinson CP, Ferris FL, 3rd, Klein RE, Lee PP, Agardh CD, Davis M, et al. Proposed international clinical diabetic retinopathy and diabetic macular edema disease severity scales. Ophthalmology. 2003;110:1677-82

7. Chandel A, Dhindsa S, Topiwala S, Chaudhuri A, Dandona P. Testosterone concentration in young patients with diabetes. Diabetes Care. 2008;31:2013-7. 
8. Caronia LM, Dwyer AA, Hayden D, Amatti F, Pitteloud N, Hayes FJ. Abrupt decrease in serum testosterone levels after an oral glucose load in men: implications for screening for hypogonadism. Clin Endocrinol. 2013;78:291-296.

9. Hall SA, Esche GR, Araujo AB, Travison TG, Clark RV, Williams RE, et al. Correlates of low testosterone and symptomatic androgen deficiency in a population-based sample. J Clin Endocrinol Metab. 2008;93:3870-7.

10. Wong SY, Chan DC, Hong A, Woo J. Prevalence of and risk factors for androgen deficiency in middle-aged men in Hong Kong. Metabolism. 2006;55:1488-94.

11. RamkishanJat, Mandavi Agarwal .Comparison Of Serum Testosterone, Luteinizing Hormone And Follicle Stimulating Hormone Levels In Diabetics And NonDiabetics Men -A Case -Control Study. Journal of Dental and Medical Sciences .2012: 65-71

12. Dhindsa S; Michael G Miller; Cecilia L McWhirter; Donald E Mager; Husam... Testosterone Concentrations in Diabetic and Nondiabetic Obese Men;Diabetes Care; 2010; 33: 1186

13. Ganesh HK, VijayaSarathi HA, George J, Shivane VK, Bandgar T, Menon PS, et al. Prevalence of hypogonadism in patients with type 2 diabetes mellitus in an Asian Indian study group. Endocr Pract. 2009; 15:513-20.

14. Janjgava S, Zerekidze T, Uchava L, Giorgadze E, Asatiani K. Influence of testosterone replacement therapy on metabolic disorders in male patients with type 2 diabetes mellitus and androgen deficiency. Eur J Med Res. 2014;19(1):56. 